21st Particles and Nuclei International Conference (PANIC 2017)

International Journal of Modern Physics: Conference Series

Vol. 46 (2018) 1860004 (8 pages)

(C) The Author(s)

DOI: $10.1142 / \mathrm{S} 2010194518600042$

\title{
Theory Concepts for Heavy Exotic Mesons
}

\author{
C. Hanhart \\ Forschungszentrum Jülich, Institute for Advanced Simulation, \\ Institut für Kernphysik and Jülich Center for Hadron Physics, \\ D-52425 Jülich, Germany \\ c.hanhart@fz-juelich.de
}

Published 3 May 2018

\begin{abstract}
Some of the currently most popular conjectures for the structure of the recently discovered heavy mesons that do not find a place in the quark model quarkonium spectrum are sketched. Furthermore, some observables are identified that should allow one to identify the most prominent components of individual states.
\end{abstract}

Keywords: Heavy quarks; exotic states.

\section{Introduction}

The observation of the $X(3872)$ in $2003^{1}$ initiated a renaissance of heavy meson spectroscopy, since the properties of this particle were in conflict with the predictions of the quark model, which was until then extremely successful in the heavy quark sector. Since then a large number of additional candidates of exotic states was discovered. What is intriguing in this context is the fact that below the first open flavor threshold the quark model continues to provide an extremely successful description of the properties of the quarkonium states - even those discovered after 2003 - while all heavy ${ }^{\mathrm{a}}$ exotics reside above this threshold. For long there was the expectation by some that with some adjustment of the quark model it is possible to describe the $X(3872)$ as a realization of the $\chi_{c 1}(2 P)$ quark model state, however, with the discovery of charged states with prominent decays into heavy quarkonia ${ }^{2}$ it became apparent that meson states beyond the most simple realization of the quark model exist. For general reviews about the heavy exotics we refer to Refs. 3, 4, 5 - reviews with specific emphasis on certain aspects are quoted in the sections below.

a'Here the notion of 'heavy exotics' is used for exotics that contain a heavy quark-(anti-)quark pair.

This is an Open Access article published by World Scientific Publishing Company. It is distributed under the terms of the Creative Commons Attribution 4.0 (CC-BY) License. Further distribution of this work is permitted, provided the original work is properly cited. 


\section{Hanhart}

The focus of this presentation is on some representative phenomenological approaches that can be found in the literature aiming at a better understanding of the newly discovered states. In general one can distinguish two classes of states beyond the naive quark model, namely those where gluonic excitations contribute to the quantum numbers, like glueballs and hybrids, and multi-quark states. In this presentation I would like to focus on the latter class. To be concrete, I will compare the phenomenology of and predictions for tetraquarks, hadro-quarkonia and hadronic molecules. In simple words these three may be distinguished by the way the heavy and light quarks arrange themselves within a given hadron: The fundamental building blocks of tetraquarks are colored heavy light diquarks and anti-diquarks, those of hadro-quarkonia are compact, colorless quarkonia, surrounded by a light quark cloud and those of hadronic molecules are pairs of heavy open flavor mesons. The phenomenological implications of these structures are discussed in some detail in the following sections.

\section{Tetraquarks}

The first detailed study of tetraquark structures dates back to the works of Jaffe employing the MIT-bag model ${ }^{6}$. Already at that time the notion of the $[\overline{3}]$ diquarks as 'good diquarks' was initiated based on the observation that in a pertrubative treatment this is the attractive channel - most of the following works therefore included only the good diquarks. While in Ref. 6 the focus was on the light quark sector, in 2003, shortly after the discovery of the $X(3872)$ in Ref. 7 the picture was adapted to the quarkonium sector. While in this original work the spin-spin interaction was dominated by that between the diquarks, in line with expectations from the heavy quark symmetry, it was realized in Ref. 8 that the $Z_{c}$ spectrum can be described only, if the spin-spin interaction acts predominantly within the diquarks. In this work it is also shown that this unusual assumption also helps to describe some decay phenomenology — in particular the transition $Y(4260) \rightarrow X(3872) \gamma$. For a recent review of multiquark states with emphasis on tetraquarks we refer to Ref. 9.

The approach starts from identifying the most relevant diquark-anti-diquark interactions contributing to the mass of a tetraquark $\mathrm{as}^{8}$

$$
\begin{gathered}
M=\hat{M}_{00}+\frac{B_{c}}{2} \vec{L}^{2}-2 a \vec{L} \cdot \vec{S}+2 \kappa_{c q}\left[\vec{s}_{c} \cdot \vec{s}_{q}+\vec{s}_{\bar{c}} \cdot \vec{s}_{\bar{q}}\right] \\
=M_{00}+B_{c} \frac{L(L+1)}{2}+a[L(L+1)+S(S+1)-J(J+1)] \\
\quad+\kappa_{c q}[s(s+1)+\bar{s}(\bar{s}+1)-3]
\end{gathered}
$$

where in the last step it was used that the Hamilonian acts on diquarks and antidiquarks with definite spin $s$ and $\bar{s}$, respectively, coupled to a given angular momentum $L$ and total spin $S$ combined to some total angular momentum $J$.

To fix the parameters one may start with the $L=0$ sector which for tetraquarks refers to even parity states. Then one is faced with three established states, namely 
$X(3872)$ - an isoscalar with $J^{P C}=1^{++}$, and $Z_{c}(3900)$ and $Z_{c}(4020)$, both isovectors with $J^{P C}=1^{+-}$which allow one to fix $\kappa_{c p}$ as well as $M_{00}$. Before one can proceed to fix the parameters that enter for $L=1$, which need to be fixed from negative parity states, one needs to decide which states to include in the fit - at present the spectrum of $1^{--}$states is not established. For example, in Ref. 10 the fit-scheme of Ref. 8, which included $Y(4008), Y(4260), Y(4360)$ and $Y(4660)$, was contrasted with an alternative scheme including $Y(4220), Y(4330)^{\mathrm{b}}, Y(4390)$ and $Y(4660)$ - in line with the most recent measurements of BESIII ${ }^{11,12}$. Furthermore the interaction was extended to allow for a spin-spin tensor force in addition to the terms mentioned above. In any case the analysis calls for 4 exotic states in the $1^{--}$sector below $4700 \mathrm{MeV}$ for the parameters to be in line with other systems. It remains to be seen how many of the states claimed at present get established eventually. Implications of Eq. (1) for other quantum numbers are discussed in Ref. 13.

It should also not remain unmentioned that there are various problems with the tetraquark picture as presented. First of all it predicts a lot more states than observed: There are not only those that follow directly from Eq. (1). The spectrum is on top doubled by the fact that all states should appear near degenerate in both the isoscalar and the isovector channel in complete analogy to the proximity in masses of $\rho$ and $\omega$. In addition it is also not clear yet, if just looking at good diquarks is appropriate $^{14}$.

Recently the interest into $Q Q \bar{q} \bar{q}$ tetraquarks, first sudied in Ref. 16, revived. There exist now studies from QCD sum rules $^{15}$, lattice QCD $^{17}$ as well as phenomenology ${ }^{18,19}$. Especially the last works employ the observation of doubly heavy baryons to make predictions for doubly heavy tetraquarks. The connection between these systems might be most compactly collected into the formula ${ }^{19}$

$$
m(Q Q \bar{q} \bar{q})-m(Q Q q) \simeq m(\bar{Q} \bar{q} \bar{q})-m(\bar{Q} q)
$$

which is based on a proposed quark-diquark symmetry ${ }^{20}$. This symmetry is realized in nature, if heavy diquarks form compact substructures in hadrons, for this would allow one to perform a systematic expansion in $r_{Q Q} / r_{q q}$, where $r_{q q}$ denotes the size of the light quark cloud that may be estimated as $1 / \Lambda_{\mathrm{QCD}}$. As soon as $r_{Q Q} / r_{q q}$ is a small parameter, one may safely assume the $Q Q$ diquark to be in the color-antitriplet configuration, since for heavy quarks the $Q Q$ interaction should be largely governed by the one gluon exchange which is attractive only in this channel. Then Eq. (2) follows naturally. Especially since recently the first doubly heavy baryon was found experimentally ${ }^{21}$, the conclusion might well be inverted: If the pattern of Eq. (2) were not realized in nature, it would tell us that QCD does not favor doubly heavy compact diquarks. Therefore the experimental search for the mentioned tetraquark structures should be performed with high priority at, e.g. Belle

\footnotetext{
${ }^{\mathrm{b}}$ This state is often referred to as $Y(4320)$.
} 
and LHCb. The above mentioned studies find typically a deeply bound $b b \bar{u} \bar{d}$ system with $J^{P}=1^{+} 100-200 \mathrm{MeV}$ below the $B^{-} \bar{B}^{* 0}$ threshold.

\section{Hadroquarkonia}

The physical picture underlying hadroquarkonia is that of a compact quarkonium core surrounded by a light quark cloud sticking together thanks to the QCD analogue of the van der Waals force ${ }^{22}$. In this approach the decay of, say, the $Y(4260)$ viewed as a $J / \psi$ core with an isoscalar pion cloud happens simply by separation of core and cloud. This provides a natural explanation, why states like $Y(4260)$ and $Y(4360)$ are seen in $e^{+} e^{-} \rightarrow J / \psi \pi \pi$ and $e^{+} e^{-} \rightarrow \psi(2 S) \pi \pi$, respectively, but not in $D^{(*)} D^{(*)}$ as expected for $\bar{c} c$ states. In order to explain the observation of both $Y(4260)$ and $Y(4360)$ in the $h_{c}(1 P) \pi \pi$ final state, where the transition to the spin 0 charmonium in the final state seems to suggest a significant amount of heavy-quark spin symmetry violation, it was proposed in Ref. 23 that $Y(4260)$ and $Y(4360)$ emerge from a mixing of a charmonium with a spin 1 core (dominated by $\psi(2 S)$ ) and one with a spin 0 core (predominantly $\left.h_{c}(1 P)\right)$.

Predictions derived from the hadro-quarkonium approach follow most straightforwardly for the partner states derived employing heavy quark spin symmetry. For example, when the $Z_{c}(3900)$ is assumed to be a hadro-charmonium composed of a $J / \psi$ core supplemented with a light quark cloud carrying the quantum numbers of the pion, then there must be a spin partner state composed of an $\eta_{c}(1 S)$ core with the same cloud ${ }^{24}$. In particular, this partner states baptized $W_{c}$ should be lighter than the $Z_{c}(3900)$ according to

$$
M_{W_{c}}=M_{Z_{c}(3900)}-M_{J / \psi}+M_{\eta_{c}} .
$$

On the contrary, if the $Z_{c}(3900)$ is a molecular state composed of $D \bar{D}^{*}$, then the spin partner should be heavier than the $Z_{c}$ by the $D^{*}-D$ mass difference ${ }^{24}$. Therefore, with the discovery of an isovector state near the $D^{*} \bar{D}^{*}$ threshold at $\mathrm{BESIII}^{25}$ the hadro-quarkonium picture is ruled out for the $Z_{c}(3900)$.

When a similar logic to identify spin partners is applied to the scenario outlined above for $Y(4260)$ and $Y(4360)$, in total 4 spin partners emerge with the distinctive feature that there emerges a relatively light $\eta_{c}(4140)$ absent from the phenomenology for the other exotic scenarios ${ }^{13}$. A more detailed comparison of features deduced from the hadro-charmonium picture and those deduced from the molecular picture for $Y(4260)$ can be found in Ref. 26.

\section{Hadronic Molecules}

Hadronic molecules are bound states of two (or more) color neutral states in close analogy to nuclei. The binding force typically comprises both short ranged interactions as well as the longer ranged exchange of the relatively light Goldstone 
bosons - especially the pion ${ }^{\mathrm{c}}$, which is regarded by many as crucial whenever it is allowed to contribute at tree level. Based on meson exchange models deuteron-like states composed of two heavy mesons were predicted already in Refs. 28, 29. For a recent review on molecular states we refer to Ref. 30 .

It is well known from nuclear physics that binding is in general the strongest in $S$-waves. Accordingly we here focus on $S$-wave systems. Furthermore, molecular states can only be formed from narrow hadrons - broad constituents would necessarily lead to broad bound states $^{31}$; the same statement from a different angle may be phrased as: The constituents must live long enough to allow the bound state to form ${ }^{32}$.

For shallow bound states Weinberg developed a criterion that allows one to extract the probability to find the molecular component in the wave function of this state, $\left(1-\lambda^{2}\right)$, directly from the residue at the bound state pole (which is the square of the effective coupling of the given state to the relevant continuum channel $)^{33}$ :

$$
\frac{g_{\mathrm{eff}}^{2}}{4 \pi}=\frac{4 M^{2} \gamma}{\mu}\left(1-\lambda^{2}\right),
$$

where $M$ denotes the mass of the bound state, $\mu=m_{1} m_{2} /\left(m_{1}+m_{2}\right)$ the reduced mass of the two constituents with mass $m_{1}$ and $m_{2}$, respectively, and $\gamma=\sqrt{2 \mu\left(m_{1}+m_{2}-M\right)}$ is the binding momentum. In Ref. 34 it was shown that this relation can still be used even in the presence of remote inelastic channels. Eq. (4) acquires corrections of the order of $\gamma R$, where $R$ denotes the range of forces. Thus, those corrections can get quite large especially for systems with heavy mesons. However, the spirit of Eq. (4) is easily generalized: ( $i)$ Transitions with the constituents in the final state are enhanced; (ii) loops that contain the constituents appear at leading order. Then, for all observables where those loops are convergent, the molecular nature of the states is expected to leave an imprint in these observables ${ }^{35}$. However, if these loops are divergent, the observable is sensitive to the short ranged structure of the wave function that is not under control in the effective field theory. This calls for a counter term at leading order - examples of observables of this class are radiative decays of $X(3872)^{36}$ or molecule production at high $p_{T}^{37}$.

Natural observables sensitive to the molecular nature are the line shapes of a given state. Those are especially non-trivial when the constituents are unstable as was first observed in Ref. 38 (see also Ref. 39). This is illustrated in Fig. 1: Shown are the experimental line shapes of $Y(4260)$ in the $J / \psi \pi \pi$ channel (first row) and the $D^{*} \pi \bar{D}$ channel (second row) compared to those that emerge from a calculation assuming a $D_{1}(2420) \bar{D}$ molecular structure for this $1^{--}$state $^{40}$ as proposed in Ref. 41. Since $D^{*} \pi$ in a D-wave is the main decay channel of the $D_{1}(2420)$ the latter channel reflects the decay of the molecule into its constituents, if indeed the state is

${ }^{\mathrm{c}}$ Note that the pion exchange potential with derivative couplings is field theoretically consistent only when accompanied by short ranged interactions ${ }^{27}$. 
Inelastic channel

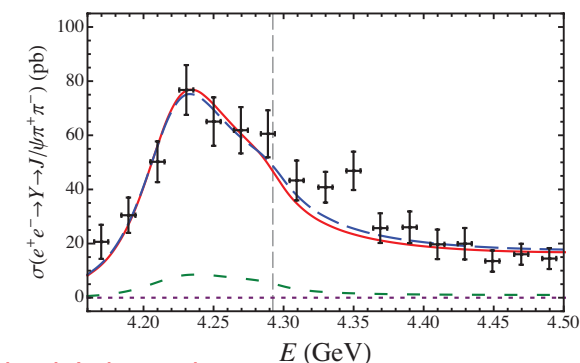

'elastic' channel

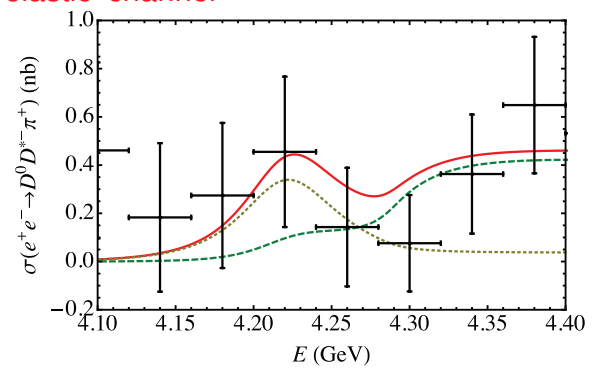

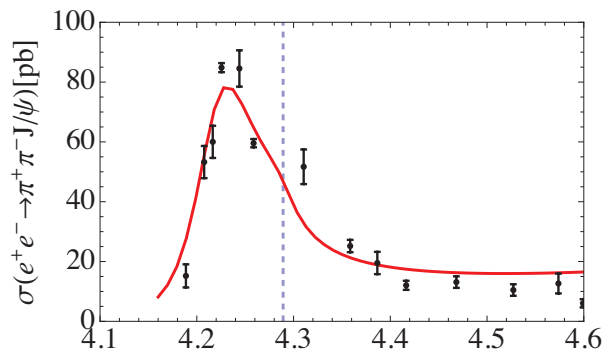

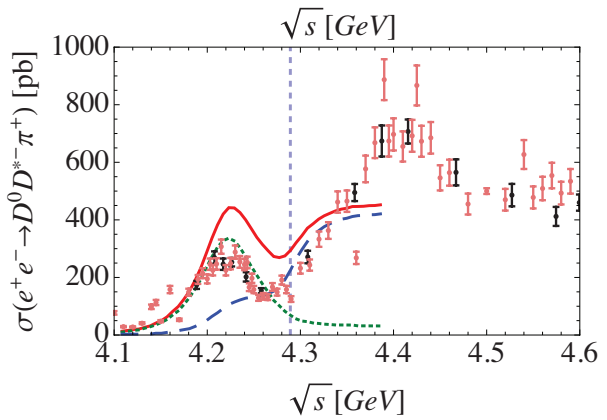

Fig. 1. The lineshapes of $Y(4260)$ as seen in both the $J / \psi \pi \pi$ (first line) and the $D^{*} \bar{D} \pi$ (second line) final states. The left panels show the data as available $2014^{42,44}$, when the parameters of the molecular model described in the text were fixed. In the upper panel left the dotted, green short-dashed, blue long-dashed and solid line refer to the background, the $Z_{c}$, the box and the full contribution. In the lower left panel the dotted, dashed and solid line refer to the $D^{*} \pi S$-wave, the $D^{*} \pi$ D-wave and the full contribution. For details we refer to Ref. ${ }^{40}$. The right panels show the previously fixed line shapes compared to the most recent data of Ref. 11 and 12 for the $J / \psi \pi \pi$ and $D^{*} \bar{D} \pi$ channels, respectively. Note that the latter data set is still preliminary.

of molecular nature. The model ${ }^{40}$ was fit to the data available in 2013 - especially the spectrum of Ref. 42, shown in the upper left panel of Fig. 1, as well as some differential spectra provided by BESIII ${ }^{43}$. In particular the angular distributions published there called for some small admixture of the $D^{*} \pi S$-wave, as shown by the dotted line in the figure - the dashed line shows the $D$-wave and the solid line the sum of both. Note that the line shape of Ref. 40 in $J / \psi \pi \pi$ peaks near $4220 \mathrm{MeV}$ and not at $4260 \mathrm{MeV}$. On the other hand a mass of $4260 \mathrm{MeV}$ was extracted from these data using a symmetric Breit-Wigner distribution. The data for $e^{+} e^{-} \rightarrow D^{*} \pi \bar{D}$ existing at the time ${ }^{44}$, shown in the lower left panel, was not included in the fit the distribution emerged as a prediction. It is therefore important to note that in the molecular picture very non-trivial line shapes emerge naturally for $Y(4260)$.

In the right column the most recent data in the $Y(4260)$ mass range for the $J / \psi \pi \pi^{11}$ and the $D^{*} \pi \bar{D}^{12}$ channel are shown together with the same curves already fixed in 2013. Obviously the gross features of the data are nicely reproduced in both channels with just one single particle state included ${ }^{40,45}$. On the contrary, based on analyses that again involved only Breit-Wigner distributions, the data shown were claimed to contain in addition to a pole at $4222 \mathrm{MeV}$ and a higher lying one $\mathrm{e}^{11,46}$. 
Besides the line shapes also the quantitative implications of spin symmetry violations turn out to be quite specific for the underlying dynamics. For the case of the spin partners of heavy meson molecules this is worked out in some detail in Refs. 47, 48, 49, 50, 51, 52 .

\section{Summary and Conclusions}

We are facing exciting times in heavy meson spectroscopy. As outlined in the presentation there is reason to hope that the upcoming high precision data from BESIII, $\mathrm{LHCb}$, Belle and BaBar as well as from the future detectors BelleII and PANDA will allow us to understand the spectrum of the newly discovered heavy meson exotics. What is especially needed to discriminate between different theoretical pictures is information about resonances in other channels as well as detailed measurements of line shapes.

Before closing a disclaimer is in order: Clearly in reality the different configurations will mix. On top of what is described here there can furthermore occur a mixing with regular mesons. Theoretical investigations of these mixings are still in their infancy ${ }^{53,54,55,56}$. However, I regard it as realistic - a judgement that might well be driven by desire - that the gross features of the properties of the exotics can be mapped onto one of the scenarios outlined in this presentation. Those studies will then eventually reveal, how (or if at all) QCD clusters its constituents into multi-quark states providing crucial insights in the inner workings of the strong interaction.

\section{Acknowledgments}

The author is grateful for the various enlightening collaborations with Vadim Baru, Martin Cleven, Feng-Kun Guo, Yulia Kalashnikova, Ulf-G. Meißner, Alexey Nefediev, Qian Wang, Qiang Zhao and Bing-Song Zou that lead to the results presented here. This work is supported in part by the DFG and the NSFC through funds provided to the Sino-German CRC 110 "Symmetries and the Emergence of Structure in QCD".

\section{References}

1. S. K. Choi et al. [Belle Collaboration], Phys. Rev. Lett. 91 (2003) 262001.

2. A. Bondar et al. [Belle Collaboration], Phys. Rev. Lett. 108 (2012) 122001.

3. N. Brambilla et al., Eur. Phys. J. C 74 (2014) no.10, 2981.

4. R. F. Lebed, R. E. Mitchell and E. S. Swanson, Prog. Part. Nucl. Phys. 93 (2017) 143.

5. S. L. Olsen, T. Skwarnicki and D. Zieminska, arXiv:1708.04012 [hep-ph].

6. R. L. Jaffe, Phys. Rev. D 15 (1977) 267.

7. L. Maiani, F. Piccinini, A. D. Polosa and V. Riquer, Phys. Rev. D 71 (2005) 014028.

8. L. Maiani, F. Piccinini, A. D. Polosa and V. Riquer, Phys. Rev. D 89 (2014) 114010.

9. A. Esposito, A. Pilloni and A. D. Polosa, Phys. Rept. 668 (2016) 1. 
10. A. Ali et al., arXiv:1708.04650 [hep-ph].

11. M. Ablikim et al. [BESIII Collaboration], Phys. Rev. Lett. 118 (2017) no.9, 092001

12. Chengping Shen, talk presented at this conference.

13. M. Cleven et al., Phys. Rev. D 92 (2015) no.1, 014005.

14. J. M. Richard, A. Valcarce and J. Vijande, Phys. Rev. D 95 (2017) no.5, 054019

15. M. L. Du, W. Chen, X. L. Chen and S. L. Zhu, Phys. Rev. D 87 (2013) no.1, 014003.

16. J. P. Ader, J. M. Richard and P. Taxil, Phys. Rev. D 25 (1982) 2370.

17. A. Francis, R. J. Hudspith, R. Lewis and K. Maltman, Phys. Rev. Lett. 118 (2017) no.14, 142001.

18. M. Karliner and J. L. Rosner, arXiv:1707.07666 [hep-ph].

19. E. J. Eichten and C. Quigg, arXiv:1707.09575 [hep-ph].

20. M. J. Savage and M. B. Wise, Phys. Lett. B 248 (1990) 177.

21. R. Aaij et al. [LHCb Collaboration], Phys. Rev. Lett. 119 (2017) no.11, 112001.

22. S. Dubynskiy and M. B. Voloshin, Phys. Lett. B 666 (2008) 344

23. X. Li and M. B. Voloshin, Mod. Phys. Lett. A 29 (2014) no.12, 145006.

24. M. B. Voloshin, Phys. Rev. D 87 (2013) no.9, 091501.

25. M. Ablikim et al. [BESIII Collaboration], Phys. Rev. Lett. 111 (2013) no.24, 242001.

26. Q. Wang et al., Phys. Rev. D 89 (2014) no.3, 034001.

27. V. Baru et al., Phys. Rev. D 91 (2015) no.3, 034002.

28. M. B. Voloshin and L. B. Okun, JETP Lett. 23 (1976) 333.

29. N. A. Tornquist, Z. Phys. C 61 (1994) 525.

30. F. K. Guo et al., arXiv:1705.00141 [hep-ph].

31. A. A. Filin et al., Phys. Rev. Lett. 105 (2010) 019101.

32. F. K. Guo and U.-G. Meißner, Phys. Rev. D 84 (2011) 014013.

33. S. Weinberg, Phys. Rev. 137 (1965) B672.

34. V. Baru et al., Phys. Lett. B 586 (2004) 53.

35. M. Cleven et al., Phys. Rev. D 87 (2013) no.7, 074006.

36. F. K. Guo et al., Phys. Lett. B 742 (2015) 394.

37. M. Albaladejo et al., Chin. Phys. C 41 (2017) 121001.

38. E. Braaten and M. Lu, Phys. Rev. D 76 (2007) 094028.

39. C. Hanhart et al., Phys. Rev. D 81 (2010) 094028.

40. M. Clevenet al., Phys. Rev. D 90 (2014) no.7, 074039.

41. Q. Wang, C. Hanhart and Q. Zhao, Phys. Rev. Lett. 111 (2013) no.13, 132003.

42. Z. Q. Liu et al. [Belle Collaboration], Phys. Rev. Lett. 110 (2013) 252002.

43. M. Ablikim et al. [BESIII Collaboration], Phys. Rev. Lett. 112 (2014) no.2, 022001.

44. G. Pakhlova et al. [Belle Collaboration], Phys. Rev. D 80 (2009) 091101.

45. W. Qin, S. R. Xue and Q. Zhao, Phys. Rev. D 94 (2016) no.5, 054035.

46. X. Y. Gao, C. P. Shen and C. Z. Yuan, Phys. Rev. D 95 (2017) no.9, 092007.

47. A. E. Bondaret al., Phys. Rev. D 84 (2011) 054010.

48. M. B. Voloshin, Phys. Rev. D 84 (2011) 031502.

49. T. Mehen and J. W. Powell, Phys. Rev. D 84 (2011) 114013.

50. J. Nieves and M. P. Valderrama, Phys. Rev. D 86 (2012) 056004.

51. V. Baru et al., Phys. Lett. B 763 (2016) 20.

52. V. Baru et al., JHEP 1706 (2017) 158.

53. G. Rupp, E. van Beveren and S. Coito, Acta Phys. Polon. Supp. 8 (2015) no.1, 139.

54. E. Cincioglu, J. Nieves, A. Ozpineci and A. U. Yilmazer, Eur. Phys. J. C 76 (2016) no. $10,576$.

55. I. K. Hammer, C. Hanhart and A. V. Nefediev, Eur. Phys. J. A 52 (2016) no.11, 330.

56. Y. Lu, M. N. Anwar and B. S. Zou, arXiv:1705.00449 [hep-ph]. 